\title{
Élise Turcotte : écrits sauvages et domestiques
}

\section{Joëlle PAPILLON, Université McMaster}

La tradition occidentale tient, de façon notoire, à l'idée d'une différence étanche entre animalité et intellectualité ; tant que l'on cherche à distinguer l'homme et à le placer à la tête des vivants ${ }^{1}$, cet écart se doit de demeurer infranchissable. L'importance de cet échafaudage intellectuel pour notre compréhension des rapports possibles entre humains et animaux a été démontrée par Jacques Derrida dans L'animal que donc je suis, où il affirme que «[r]ien n'est plus détestable, [...] plus haïssable, plus odieux [...] à l'homme [...] que le souvenir d'une ressemblance ou d'une affinité entre l'homme et l'animalité » (142). La lecture déconstructionniste de textes philosophiques majeurs (entre autres ceux de Descartes, Kant et Heidegger) mène Derrida au constat de la dénégation profondément enracinée du regard des animaux (28-32), de la diversité animale (64-65) et de leur faculté de réponse (54; 163-191). Heureusement, plusieurs écrivaines et écrivains se sont révélés capables de prendre en compte cette diversité des vivants, leur regard et leur réponse, notamment l'auteure québécoise Élise Turcotte, dont les écrits récents ${ }^{2}$ sont peuplés de compagnons non humains - en premier lieu de chats et d'oiseaux. L'une de ses narratrices envisage son rapport aux animaux comme un rapprochement progressif, où c'est elle qui se trouve apprivoisée: "ils sont d'abord venus habiter des poèmes, puis des pages de romans, pour ensuite entrer dans mon jardin, et puis ma maison » (Pourquoi, 32). Dans ce passage, le sujet féminin ne fait aucun geste pour s'approprier l'animal - le prendre au piège ou le dompter - préférant adopter une attitude d'ouverture et de disponibilité qui permet la rencontre et la relation. Turcotte et ses personnages sont fascinées par la dimension insaisissable des animaux: même familiers, domestiques, les animaux nous demeurent étrangers, ce dont elle prend conscience chaque fois qu'elle rencontre le regard d'un animal.

Son traitement des relations entre humains ${ }^{3}$ et animaux est particulièrement intéressant dans deux cuvres discontinues, Pourquoi faire une maison avec ses morts (2007) et Autobiographie de l'esprit (2013) - ainsi que dans le roman La maison étrangère (2002), sur lequel je reviendrai en conclusion. La première est un recueil de récits fictifs revisitant les questions de la mort, de la catastrophe et du deuil, tandis que la seconde se présente comme un 
collage de réflexions sur l'écriture, de souvenirs d'enfance, de rêves, d'obsessions et de quelques photos. D'un texte à l'autre, l'on retrouve une femme vivant avec deux enfants, quelques chats et un oiseau, les animaux semblant faire partie du portrait de famille et jouer un rôle important dans la cohésion de la maisonnée. Les œuvres de Turcotte convoquent un univers assez particulier, qui allie le quotidien domestique somme toute banal à un discours intellectuel parfois étouffant. C'est que, chez Turcotte, vie de l'esprit et vie du corps ne sont pas tenues pour des contraires ou des empêchements l'un à l'autre, mais bien comme des aspects de l'expérience impossibles à dénouer. C'est peut-être même l'une des choses qui intéressent le plus Turcotte : le lien de continuité qui unit les êtres entre eux, ainsi que l'esprit au corps et la vie à la mort.

Cela est particulièrement visible dans Autobiographie de l'esprit, où des figures animales surgissent presque chaque fois qu'elle parle d'écriture. Elle se représente le livre en devenir comme un espace peuplé de créatures indomptées, un monde excitant et dangereux, dont elle prétend «essa[yer] d'apprivoiser la sauvagerie » (139). Il me semble toutefois que Turcottte ne reprend pas du tout la dynamique hiérarchique souvent associée à l'apprivoisement (où l'humain « civilisé » domestique un animal sauvage et le rend servile), entre autres parce qu'elle prend soin de respecter l'espace sauvage d'autrui - que cet autre soit humain ou non. Notons également que Turcotte s'inscrit elle-même dans cet espace ambigu entre le sauvage et le domestique en affichant son moi d'écrivaine dans des images animalières. Bien que ce soit parfois pour exprimer une angoisse - lorsqu'elle est un «papillon de nuit virevoltant dans un bocal, affolée par le livre à venir » (14) -, ces autoportraits d'écrivaine en animal surgissent surtout dans les moments où l'écriture est vécue comme une évidence calme - elle dit par exemple que les mots essaiment devant ses yeux (46) ou lui apparaissent «aussi clairement qu'un troupeau d'élans à l'entrée d'une vallée » (23) - ou au contraire comme une ivresse : quand les mots arrivent « comme des panthères qui courent» (49), ses mains qui écrivent «galopent comme un cheval non apprivoisé à travers la ville où le feu a pris » (74). Il est intéressant de remarquer que, pour se représenter écrivant, elle se tourne exclusivement vers des images d'animaux sauvages qui fuient les liens du domestique, tandis que les personnages animaux qu'elle développe dans ses écrits sont généralement des animaux familiers: d'un côté, les animaux sauvages qui s'échappent vers le lointain, qui traversent les pages sans s'y arrêter; de l'autre, les animaux domestiques qui côtoient les personnages féminins, avec qui une relation peut être développée. Bref, les animaux s'invitent sans cesse dans ses pages, aussi bien comme autre qui lui retourne 
son regard que comme figures du soi de l'écrivaine. Deux de leurs fonctions m'intéresseront dans les pages qui suivent : l'accompagnement et la consolation, ainsi que la représentation de soi.

\section{Accompagnements}

Dans l'œuvre de Turcotte, les animaux sont avant tout « des compagnons de vie » et des «guérisseurs de solitude» (Pourquoi, 32), mais il serait plus juste d'établir que tous les partenaires de la relation (humains aussi bien qu'animaux) ont à la fois à guérir eux-mêmes d'une solitude et à guérir la solitude de l'autre, puisque les animaux sont présentés comme initialement seuls, perdus, fragiles, dans des situations précaires, puis «sauvés » par une adoption ou un apprivoisement ${ }^{4}$. De même, les personnages de femmes et d'enfants, blessés ou souffrants ${ }^{5}$, trouvent dans leur relation avec des animaux domestiques un réconfort salvateur comme si, pour continuer à vivre, il leur fallait pouvoir prendre soin d'un autre vivant. Dans une étude sur les représentations de l'espace américain, Marie Parent propose que la narratrice de Pourquoi faire une maison avec ses morts tente « de recréer une forme de cohésion entre les individus, en devenant [...] le centre d'une communauté d'،accidentés'» (123). Bien qu'une telle lecture soit tout à fait juste, il faudrait ajouter les animaux recueillis à cette communauté d'accidentés qui, en s'appuyant les uns sur les autres, cherchent tant bien que mal à recoller les morceaux d'une société en miettes ou à trouver le moyen de survivre à une époque violente et chaotique. L'une des nouvelles, par exemple, dépeint les difficultés de l'existence quotidienne dans un monde post-apocalyptique ; un matin, un «miracle » se produit : la narratrice se réveille dans un état de 'consolation inexplicable' et se rend compte qu' « un petit chat était en train de lécher les larmes qui coulaient sur [ses] joues » (105). Lorsque le souvenir douloureux de son père défunt l'attriste, la narratrice d'un autre récit est réconfortée par un chat qui se presse sur ses jambes ; elle le déclare «clairvoyant» (24) parce qu'il a su reconnaître qu'elle avait besoin de lui. Dans ces deux exemples, l'accompagnement animal passe avant tout par le toucher, puisqu'un animal vient caresser la femme, en une inversion du rapport domestique stéréotypé (selon lequel l'humain caresse «son » animal domestique) mettant l'accent sur l'agentivité de l'animal - étant donné qu'il est l'initiateur du rapprochement. Cependant, le contact physique n'est pas le seul type d'interaction entre humains et animaux chez Turcotte, comme le montre la 
relation complexe développée entre un oiseau et sa famille d'adoption dans une autre nouvelle dont l'analyse suit.

Dans ses œuvres, Turcotte met en scène de façon répétée à la fois la compassion d'une femme pour un animal souffrant et celle d'un animal pour une femme souffrante ${ }^{6}$, mais la compassion éprouvée par l'animal mène souvent à son sacrifice, puisqu'il se met à porter la souffrance familiale. Consciente que l'apparence heureuse de sa famille n'est qu'une illusion fragile qui menace de s'écrouler à tout instant (pour des raisons qui ne sont pas explicitées dans le texte), l'une des narratrices de Pourquoi faire une maison avec ses morts cherche à alléger l'atmosphère. Elle pense que de vivre avec un animal pourrait les rassembler en un projet commun et les « humaniser»:

nous ne savions pas comment faire pour approcher les êtres. Rien, à ce moment, même pas un galet ramassé sur la grève, même pas un chien sans volonté, n'aurait pu être apprivoisé par nous. Nous nous trouvions déjà, sans le savoir, sur la pente conduisant à un cruel enfermement. Voilà pourquoi j'ai cru, malgré notre bonheur apparent, qu'un petit animal nous était nécessaire. Il était urgent de commencer à faire vivre d'autres espèces dans cette maison. (33)

Le commentaire sur l'incompétence supposée de la famille en ce qui a trait à l'apprivoisement est des plus intéressants : les membres de la famille seraient-ils si peu attachants que pas même un chien ne chercherait à les approcher, ou bien serait-ce plutôt que l'habilité d'être présents à l'autre manque tout simplement à ces humains, eux qui ne sauraient pas même s'arrêter à un galet pour le détacher de l'anonymat et s'y attacher? En adoptant un animal, la narratrice cherche, semble-t-il, à remédier à une carence affective troublante qu'elle perçoit chez ses proches ; prendre soin d'un animal, l'aimer, est présenté comme une voie de sortie de la solitude qui mine l'unité familiale.

L'oiseau adopté semble d'abord confirmer les craintes de la narratrice et résister à son rôle, puisqu'il refuse d'apprendre des tours ou d'imiter des bruits comme les autres perruches, ce que la famille perçoit comme un échec dont elle est en partie responsable en raison de sa détresse et de son incompétence. En fait, si la perruche résiste, c'est plutôt, selon moi, que la famille s'est initialement trompée sur la fonction de l'oiseau. La femme et les enfants se sentent rejetés par son refus de participer à l'échange qu'ils proposent, mais l'oiseau rectifie la relation : il n'est pas là pour les faire rire ou les impressionner par ses prouesses, mais bien pour les réconforter et les accompagner dans leur chagrin - ce qu'il fera à sa propre façon et de son propre chef. Quand le malheur diffus présent dans la maisonnée culmine en un abandon amoureux, la narratrice se 
retrouve seule avec les enfants et l'oiseau. Dès que les enfants sont partis à l'école, elle se met à pleurer, ce qui provoque ce qu'elle décrit comme un « miracle»:

l'oiseau est sorti de sa cage et est venu se poser sur mon épaule tandis que je pleurais. J'ai continué à pleurer, [...] mais l'oiseau est demeuré perché sur moi. [...] je me suis accrochée [...] à l'idée que l'oiseau était venu me consoler. [...] Chaque matin, ensuite, l'oiseau semblait attendre la musique de mes plaintes pour se poser sur mon épaule. [...] une douceur anormale accompagnait mon désespoir. C'était doux et grave. C'était la fin de notre monde, mais des fleurs poussaient sur la terre désertée. (40)

L'oiseau, en frottant son bec sur les cheveux de la narratrice, accompagne sa douleur et lui permet de faire son deuil de l'homme qui l'a quittée. Tandis qu'elle doit composer un masque de normalité et de gaieté devant ses enfants, elle n'a rien à dissimuler dans son rapport à l'oiseau' Ce geste de rapprochement initié par la perruche porte à reconsidérer le premier contact entre la famille et l'oiseau : les humains tentent d'abord d'imposer un type de relation à la perruche ; celle-ci refuse de s'engager dans cette relation, mais elle en propose une autre, qui se révèle parfaitement adaptée aux besoins de la femme - comme si l'oiseau avait d'abord eu besoin de l'étudier afin de déterminer la réponse la plus adéquate à son chagrin.

Les mois passent, et la femme est en voie de guérison, mais l'oiseau tombe malade ; c'est un cancer, il va mourir. L'angoisse et la souffrance retombent sur la maisonnée ; la narratrice n'arrive pas à y croire : « un cancer chez un si petit être vivant. [...] Ça n'avait pas de sens. Ils ont les mêmes maladies que nous, m'avait expliqué le vétérinaire. Notre espace. Nos habitudes. Nos maladies » (42). L'horreur de l'agonie de l'oiseau est amplifiée du fait que la femme s'en sent responsable, comme s'il aurait été dans l'ordre des choses qu' elle développe la maladie, et non un oiseau innocent. Le commentaire du vétérinaire laisse entendre qu'au contact de l'humain, les animaux familiers développent des maladies dont ils auraient autrement été épargnés, comme si nous étions une présence toxique dans leur existence. À la douleur de la famille endeuillée de l'oiseau s'ajoute une culpabilité diffuse, comme si l'oiseau avait offert sa mort pour préserver la famille d'une plus grande perte - la perruche avait «pris [leur] maladie sous son aile » (43). Les réactions des membres de la famille à l'annonce de la mort imminente de l'oiseau montrent bien l'importance que celui-ci avait pris parmi eux : les deux enfants sont inconsolables et le fils crie qu'il « l'aime plus que n'importe quel humain » (42). Perplexe devant cette douleur qu'il trouve démesurée, le grand-père (qui ne vit pas avec eux dans la maison) dit à sa petite-fille de cesser de pleurer : «c'est juste un oiseau, vois-tu, imagine ma mort à moi, ou 
celle de ta mère » (42), ce que la narratrice commente de la façon suivante : «Ces paroles étaient si déplacées, [...] si étranges en fait, que j'ai pensé [...] qu'il avait un instant perdu la raison » (42). Pour les proches de l'oiseau, l'idée qu'il y ait une hiérarchie - des morts (humaines) qui comptent et d'autres (animales) qui ne comptent pas - est absolument inacceptable et même, pour la mère, un signe de folie. Les paroles maladroites du grand-père sont obscènes dans le contexte du deuil de la perruche, parce qu'elles signalent son échec à envisager le statut de l'animal au sein de la famille (statut qui demeure inimaginable pour lui) ${ }^{8}$ : « Il n'est pas qu'un oiseau, a repris ma fille. Je le savais bien. Il était notre petit sauveur » (42; italiques dans l'original).

Si le récit nous fait comprendre que la perruche est venue accompagner la narratrice et ses enfants lors d'une période de souffrance aiguë et qu'elle s'est sacrifiée ${ }^{9}$ pour leur épargner un plus grand mal, Turcotte laisse entendre qu'un autre accompagnement tout aussi important a eu lieu. À l'animalerie, l'oiseau a tout fait pour séduire la narratrice et son fils :

nous avions choisi cette petite perruche [...] parmi des dizaines d'oiseaux piaillant pour sortir de leur cage. La petite perruche verte semblait plus agile que les autres. Son plumage était plus brillant. Elle nous appelait, nous l'avons su tout de suite. J'ai compris plus tard [...] qu'elle était trop âgée, peut-être même déjà malade [...]. Celle-là, notre petite perruche, avait mis ses plus beaux atours pour nous suivre. Elle voulait sans doute, de ça je suis certaine maintenant, mourir parmi nous. Elle nous avait choisis, sentant de son âme sauvage que nous avions besoin de sa mort. Elle, qui allait bientôt s'en aller, pourrait emporter ce qui n'en finissait plus de mourir dans la maison. (33-4)

Non seulement la narratrice reconnaît-elle à l'oiseau la faculté de développer des stratégies de séduction dans ses rapports aux humains, mais elle accepte d'avoir été dupe du subterfuge, reconnaissante d'avoir été choisie par la perruche comme accompagnatrice de sa mort. Lorsque l'oiseau agonise, la narratrice caresse ses plumes en un retour du geste que l'oiseau avait eu pour elle, et le réconforte de sa présence jusqu'à la fin. Toutes deux souffrantes, femme et perruche se sont accompagnées et réconfortées. En fait, quand Derrida s'arrête à la question posée par le philosophe Jeremy Bentham au sujet des animaux («Can they suffer?»), il propose que «pouvoir souffrir» est précisément ce qu'humains et animaux ont en commun: «Pouvoir souffrir [...]. Là se loge, comme la façon la plus radicale de penser la finitude que nous partageons avec les animaux, la mortalité qui appartient à la finitude même de la vie, à l'expérience de la compassion, à la possibilité de partager la possibilité de cet im-pouvoir, [...] [et] l'angoisse de cette vulnérabilité » (49). C'est aussi il me semble ce que dit Donna Haraway 
dans When Species Meet : on ne peut pas éliminer la souffrance animale, mais, éthiquement, on doit la reconnaître, la minimiser et l'accompagner (83). Cet aspect des relations entre espèces est bien visible dans les œuvres de Turcotte, où personnages féminins et animaux se rejoignent dans une même pratique d'accompagnement dans la souffrance: partager la douleur, c'est déjà l'alléger. Pourquoi faire une maison avec ses morts place donc humains et animaux en relation sous le signe de la réciprocité et de la compassion.

\section{L'animal e(s)t moi}

La philosophe Elisabeth de Fontenay s'interroge sur le lien entre compassion pour la souffrance animale et identification avec les animaux en commentant un passage d'un roman de Karl Philipp Moritz où un personnage masculin observe de près du bétail sur le point d'être mis à mort, jusqu'au moment où il cesse de voir toute différence entre ces animaux et lui : "Qu'il suffise de dire que ce troublant exercice de proximité, cette obsession d'empathie et d'identification, [...] semble pouvoir conduire à la plus profonde pitié » (1011). Différents rapprochements entre femmes et animaux sont à l'œuvre chez Turcotte, mais il est impossible d'y ignorer ce glissement souligné par Fontenay où la compassion éprouvée pour un animal souffrant mène à l'identification avec lui. Soulignons toutefois que dans l'exemple donné par Fontenay, l'homme se force à l'exercice de la proximité avec les animaux parce qu'il cherche à comprendre ce qui le sépare d'eux ; chez Turcotte, la proximité avec les animaux n'est jamais forcée et ne relève pas d'un effort intellectuel. Bien plutôt, il s'agit d'un rapport affectif avec un significant other dans l'acception que Haraway (Manifesto) donne à cette expression. Haraway utilise l'expression « significant other » (littéralement l'« autre significatif » mais figurativement le partenaire ou la compagne) pour parler des relations unissant les «espèces de compagnie » (companion species), par exemple une femme et la chienne avec qui elle vit. Contrairement à sa traduction française ${ }^{10}$, l'expression significant other préserve l'altérité du partenaire (other) tout en soulignant son importance pour le sujet (significant), dont le rapport au monde dépend en partie de sa relation avec cet « autre significatif ».

L'Autobiographie de l'esprit de Turcotte s'ouvre sur deux photos collées l'une contre l'autre, sans titre et sans commentaire; sur l'une, l'on reconnaît l'auteure enfant alors que l'autre

représente un jeune cerf. À la page suivante, elle mentionne que ses enfants lui ont donné un «nom totémique », «cerf aux yeux verts »(13), ce qui nous encourage à lire ces photos 
juxtaposées comme un seul autoportrait : moi, l'animal. L'association entre Turcotte et le cerf remonte à l'enfance, alors qu'elle est tombée face à face avec un chevreuil mort, un matin qu'elle se promenait seule sur le bord de la mer. Elle raconte qu'elle est passée de l'incrédulité à l'horreur, de la fascination à l'identification: «Je sais que je suis ce chevreuil. Ma transformation a commencé là, sur la plage, à mon insu. Et je porte des bois, maintenant. Je bouge comme un animal aux aguets, cou tendu, mes yeux fixent l'humanité derrière un rideau d'arbres » (181). La métamorphose invisible du corps de la narratrice l'aide à mieux percevoir son rapport au monde et à l'autre : devenir un animal aux aguets, c'est adopter une posture de retrait. Elle peut fixer l'humanité derrière un rideau d'arbres parce qu'elle s'en est dissociée, la regardant de loin, comme quelque chose de suffisamment menaçant pour qu'il faille demeurer aux aguets, prête à détaler. À différentes reprises dans les deux œuvres de Turcotte à l'étude, elle mentionne d'ailleurs le tableau Le cerf blessé de Frida Kahlo ${ }^{11}$, dans lequel elle dit reconnaître une image de sa douleur. Poser son propre visage sur le corps du cerf blessé, c'est partager avec empathie la souffrance de l'animal et la mettre sur le même plan que la souffrance humaine comme les enfants l'avaient fait spontanément avec la mort de la perruche (dans la nouvelle étudiée plus haut) qui, pour eux, était une « vraie mort $»^{12}$.

Il n'est pas indifférent que le cerf dont Turcotte se sent soudain si proche, soit mort, et sa première pensée est qu'il s'est peut-être suicidé ${ }^{13}$. Le face-à-face avec l'animal mort provoque un profond sentiment de détresse et de pitié chez la narratrice, et l'identification avec le cerf lui permet de donner voix à cette douleur qui l'assaille. Rappelons que Fontenay définit la pitié comme « une contemplation désolée de la souffrance »(1017) mais aussi comme «une extase qui arrache à l'identité et au propre »(1018), d'où la possibilité d'identification à l'autre souffrant. Les animaux malades ou morts sont nombreux chez Turcotte, mais chacun est accompagné, soigné, cajolé, puis pleuré :

je trouve un petit chat malade, il se colle dans mon cou, sur mes cuisses, mon cœur, mes cheveux, je le soigne, le nourris, ses yeux sont presque disparus sous leur troisième paupière, il passe la nuit suivante dans une boîte, endorloté de couvertures, emmailloté avec son nouveau nom, au lever, je l'emmène chez le vétérinaire, la liste de ses maladies est longue, et il meurt tandis que je m'effondre en pleurant dans le stationnement, l'euthanasie est gratuite pour moi, merci, je pleure beaucoup trop pour un petit chat, un inconnu qui plus est, je pleure trop fort, je suis priée de sortir du monde, un chat de ferme, mais je pleure, je rêve de son corps, je perçois toujours la maigreur de ses côtes, [...] je repasse des années entières, tout ce que je n'ai pas réussi à sauver (Autobiographie, 72-3). 
Le nombre effarant de scènes similaires, surtout avec des chats et des oiseaux - et bien sûr le cerf -, laisse croire que les animaux recherchent la narratrice pour mourir auprès d'elle. Les personnages féminins de Turcotte sont plongés dans le deuil à chaque mort d'un animal, même inconnu, ce qui est condamné par les gens autour d'elles comme un excès rebutant, une marque de folie. Du point de vue des narratrices, toutefois, c'est l'absence d'empathie pour la souffrance et la mort animales, le sentiment que cette souffrance et ces morts ne nous regardent pas, nous demeurent étrangères au point de ne pas constituer une souffrance et une mort réelles, qui sont désignés comme incompréhensibles, inhumains. Pour elles, ce n'est jamais juste un chat, un oiseau ou un cerf quelconque, mais toujours un chat, un oiseau ou un cerf en particulier, un chat, un oiseau ou un cerf qui les a choisies et accompagnées dans une expérience singulière, même si ce n'était qu'en mourant. Derrida insiste d'ailleurs sur la distinction entre parler de l'animal et parler des animaux : rejeter la diversité animale derrière un substantif singulier et abstrait permet aux philosophes de ne pas voir les expériences particulières des animaux et, par là, évacue la question de la compassion (54).

Enfin, une dernière figure souffrante obsède l'écrivaine : la sirène du conte d'Andersen. Turcotte s'identifie également à la petite sirène, tenue à l'écart du monde qu'elle désire rejoindre, prête à de grands sacrifices pour réaliser un rêve qui demeure malgré tout inatteignable. La sirène est bien sûr déjà une figure hybride - mi-femme, mi-poisson -, mais dans Autobiographie de l'esprit, Turcotte s'approprie cette figure en l'hybridant davantage : elle lui ajoute des bois de cerf, venant en quelque sorte «signer» le personnage du conte et réaffirmer sa dimension animale $^{14}$. La petite sirène est un personnage intéressant, dans la mesure où elle traverse des espaces interdits, elle est celle qui aura connu les deux royaumes - celui où l'on nage, celui où l'on marche les pieds percés par la douleur de mille aiguilles -, et elle est celle qui aura aimé jusqu'à accepter de tout perdre. Se représenter en écrivaine grâce à cette sirène à bois de cerf place Turcotte dans l'entre-deux mondes et insiste sur son désir de rapprochement et d'identification aux animaux. La sirène, le cerf à visage humain, ainsi que les autres figures hybrides convoquées par Turcotte font preuve de l'exploration des limites séparant humains et animaux; dans ce collage, les éléments que l'on croyait être irrémédiablement étrangers se voient réconciliés, puisqu'ils peuvent cohabiter en un même être.

L'on pourrait concevoir ces figures hybrides dans lesquelles les contraires ne s'opposent plus comme l'illustration d'une tension qui traverse son œuvre. Dans le roman La maison 
étrangère (2002) dont il serait également intéressant de discuter, l'amant de la narratrice assemble un bestiaire à partir de photos d'animaux qui révèlent de nouvelles dimensions de chacun d'entre eux. Au départ, la narratrice est surtout frappée par les images d'animaux rares comme le dragon de mer feuillu, qu'elle décrit comme «spectaculaire » (172) et qui représente pour elle un oasis de beauté dans un monde en marche vers la destruction (173). Mais une fois les images assemblées, celles qui la marquent le plus sont les représentations d'animaux familiers ${ }^{15}$. Dans ce qu'elle nomme des «portraits », on « voyait la tête d'un animal en plan très rapproché, le regard fixé sur l'objectif. [...] Cette partie du Bestiaire [...] était [...] composée en grande partie d'animaux [...] domestiques [...], mais ils étaient photographiés de telle façon qu'on avait l'impression d'en découvrir à la fois l'aspect sauvage et familier» (178). Ce commentaire sur la dimension double des animaux rappelle le sous-titre d'Autobiographie de l'esprit, «Écrits sauvages et domestiques »; si l'on pourrait d'abord considérer le et comme l'addition de deux réalités différentes (des écrits sauvages et des écrits domestiques), je serais plutôt tentée de l'interpréter comme la marque d'une fusion entre ces deux termes seulement antinomiques de façon superficielle : chez Turcotte, les animaux familiers - comme les écrits - sont à la fois sauvages et domestiques. Vers la fin de L'animal que donc je suis, Derrida dit du chat qu'il est «un animal domestique, mais [...] non apprivoisé, non dressé, non 'domestiqué' »(214). Turcotte étendrait sans aucun doute cette description aux chats et aux oiseaux qui l'entourent en parfaits compagnons - c'est-à-dire assez proches mais toujours indépendants.

Elle utilise d'ailleurs rarement la forme possessive - mon chat, mon oiseau - pour désigner les animaux avec qui elle vit, ce qui reconnaît à l'animal une autonomie et une dimension d'étrangeté. Turcotte ne se situe pas dans un registre de l'appropriation, mais bien dans une «esthétique de la proximité » comme le proposait François Paré dans une étude sur sa poésie (38). Être proche - par le contact affectueux, par le regard échangé - est aussi faire preuve d'attention vis-à-vis de l'autre animal, c'est-à-dire à la fois d'intérêt, de respect et de soins ${ }^{16}$. Il me semble que, par là, Turcotte se rapproche de ce que le poète de la nature Don McKay appelle l'attention poétique: «there is a state of mind which I'm calling «poetic attention » [...] : it's a sort of readiness, a species of longing which is without the desire to possess [...]. To me, this is a form of knowing which counters the 'primordial grasp' in home-making, and celebrates the wilderness of the other ; [...] this kind of knowing remains in touch with perception » (26-27). Cette attention poétique à l'animal devant soi est une pratique de l'humilité et du respect : on 
reconnaît à l'autre son unicité et son espace de mystère. L'attention poétique me semble aller de concert avec la pensée de Haraway qui nous enjoint à nous demander « qui est cet animal devant moi ? $\gg^{17}$, à être attentifs à nos compagnons animaux (qu'est-ce qu'ils aiment ? comment jouentils ?) en les regardant comme des individus uniques qu'il faut prendre le temps de découvrir. Dans The Companion Species Manifesto, Haraway réfléchit aux rapports multiples et complexes qui unissent les chiens et les humains. Elle note que cette relation présente un défi particulier : «In short, the major demand on the human is precisely what most of us don't even know we don't know how to do - to wit, how to see who the dogs are and hear what they are telling us, not in bloodless abstraction, but in one-on-one relationship, in otherness-in-connection » (45). Un obstacle majeur au développement de cette relation réelle avec un animal est la persistance de la croyance en un exceptionnalisme humain - c'est-à-dire une séparation radicale entre humains et animaux, marquée par des rapports hiérarchiques qui empêchent une réelle attention de l'humain envers l'animal et donc la communication entre les espèces. Pour Haraway, l'attention sincère à l'autre est essentielle à l'établissement de toute relation : «all ethical relating, within or between species, is knit from the silk-strong thread of ongoing alertness to otherness in relation » (50). À ce titre, l'identification pourrait être considérée comme un geste vers l'animal, surtout si elle se cristallise dans des figures hybrides où l'animal et l'humain demeurent tous les deux visibles, dans une assimilation imparfaite.

Pour en revenir au bestiaire décrit dans La maison étrangère, la technique du plan rapproché cadre les animaux de façon à ce qu'il soit impossible de leur dénier un visage, ce qui cause une révélation chez la narratrice. Fontenay souligne à quel point cette question de l'existence d'un visage est importante puisque, chez Levinas, «c'est la transcendance du prochain qui s'exprime dans le visage, en ce qu'il rappelle l'interdiction de tuer à celui qui le regarde et qu'il réveille sa responsabilité » (Fontenay 953). Fontenay poursuit en affirmant que c'est «l'absence de visage, chez l'animal, qui autorise donc [...] que continuent de se perpétrer des mises à mort auxquelles est dénié en toute bonne conscience le statut de meurtre » (954). Nulle trace de ce déni chez Turcotte, où les animaux ont résolument un visage, une intériorité et par là un mystère. ${ }^{18}$ Le plan rapproché des photographies du bestiaire met également l'emphase sur le regard que les animaux nous adressent, et rappelons que Derrida amorçait sa réflexion sur la question du regard animal : «[1'animal] peut se laisser regarder, sans doute, mais aussi, la philosophie l'oublie peut-être, elle serait même cet oubli calculé, il peut, lui, me regarder. Il a 
son point de vue sur moi » (28). Turcotte mentionne plusieurs fois sa propre fascination pour les toiles de Théodore Géricault représentant des chevaux ; ce qui l'obsède dans ces images, c'est précisément le retour du regard : «enfin proche, enfin seule avec eux. Je suis dans la vision, rien, tout, la vue, dans les yeux noirs de ces chevaux qui regardent »(Autobiographie, 138). Mais ce regard demeure empli de mystère et d'ambiguïté : qu'ont à dire les animaux qui vivent avec nous, parmi nous, et que peut-on leur répondre ? Qui sont-ils, et que sommes-nous à leurs yeux ? Turcotte adopte la perspective de l'attention poétique afin de préserver un espace sauvage respectant l'inconnaissable chez l' « autre significatif », tout en créant un espace domestique avec ce qui est connaissable lorsque l'on fait l'effort de la relation avec «l'autre significatif », et cela pour chaque être qui surgit dans ses pages.

\section{Bibliographie}

Brassard, Denise. «Entretien avec Élise Turcotte ». Voix et Images 31.3 (2006) : 15-30.

Derrida, Jacques. L'animal que donc je suis. Paris : Galilée (La philosophie en effet), 2006.

Duras, Marguerite. Écrire. Paris : Gallimard, 1993.

Flaubert, Gustave. «Un cœur simple». Trois contes. Paris : Gallimard (Folioplus classiques), [1877] $2003: 9-57$.

Fontenay, Elisabeth de. Le silence des bêtes : la philosophie à l'épreuve de l'animalité. Paris : Fayard (Points-Essais), 1998.

King, Thomas. The Truth About Stories : A Native Narrative. Toronto : House of Anansi P (CBC Massey Lectures Series), 2003.

Haraway, Donna. The Companion Species Manifesto : Dogs, People, and Significant Otherness. Chicago : Prickly Paradigm P, 2003.

---. Manifeste des espèces de compagnie. Chiens, humains et autres partenaires. Tr. Jérôme Hensen. Paris : Terra Incognita / Éclat, 2010.

---. When Species Meet. Minneapolis : U of Minnesota P (Posthumanities), 2008.

McKay, Don. Vis à Vis : Field Notes on Poetry \& Wilderness. Wolfville : Gaspereau P, 2001.

Nouët, Jean-Claude. «Protection ou respect de l'animal ? ». Si les lions pouvaient parler : essais sur la condition animale. Dir. Boris Cyrulnik. Paris : Gallimard, 1998.

Paré, François. «Pluralité et convergence dans la poésie d’Élise Turcotte ». Voix et Images 31.3 (2006) : 35-45. 
Parent, Marie. «Le corps accidenté chez Hélène Monette et Élise Turcotte : l'Amérique des lieux clos ». Voix et Images 37.1 (2011) : 115-128.

Turcotte, Élise. Autobiographie de l'esprit: écrits sauvages et domestiques. Montréal: La Mèche (L'Ouvroir), 2013. ---. La maison étrangère. Montréal : Leméac, 2002. ---. Pourquoi faire une maison avec ses morts. Montréal : Leméac, 2007.

\section{NOTES}

${ }^{1}$ Il va sans dire qu'il s'agit là d'une conception du monde qui n'est pas partagée par l'ensemble des individus ni des cultures. L'écrivain cherokee Thomas King contraste par exemple l'un des récits de la création amérindiens à celui présent dans la Genèse : tandis que dans l'histoire de « la Femme tombée du ciel », le monde est créé par une série de coopérations entre humains et animaux, celle de la Bible célèbre une suite de hiérarchies (Dieu, l'homme, la femme, les animaux) (King 23-24). King conclut à propos du récit chrétien : «Unfortunately, by the time we arrived in the wilderness, broke and homeless, the story of being made in God's image, of living in paradise, of naming the animals must have gone to our heads, for while we weren't the strongest or the fastest or the fiercest creatures on the planet, we were, certainly [...], the most arrogant» (28). Au sujet de l'arrogance humaine et de son impact sur l'existence animale, voir l'article « Protection ou respect de l'animal ? » de Jean-Claude Nouët.

${ }^{2}$ En 2006, Turcotte remarque en entretien que les animaux se font de plus en plus nombreux dans ses livres (Turcotte à Brassard 20). Elle propose qu'ils lui permettent peut-être de regarder la mort telle qu'elle est (24), puisqu'ils ont avec celle-ci un rapport très différent de celui développé par les humains. Comme nous le verrons plus loin, chez Turcotte, les rapports humains/animaux sont souvent marqués par la mort, que ce soit parce que la narratrice est témoin de la mort d'un animal ou parce qu'un animal vient la consoler de la disparition d'un proche.

${ }^{3}$ Les humains mis en scène dans les œuvres de Turcotte sont très majoritairement des femmes et des enfants, dont on peut supposer que le rapport affectif aux animaux diffère au moins en partie de celui de l'homme blanc hétérosexuel stéréotypé. Turcotte mentionne en entretien son désintérêt pour les personnages masculins : "On m'a également fait remarquer qu'il n'y avait pas d'hommes dans mes livres, qu'ils étaient tous effacés. Et alors ? Qu'estce que ça peut faire ? » (Turcotte à Brassard 16). Dans l'œuvre de Turcotte, l'« Autre » au contact de qui les personnages féminins construisent leur subjectivité est plus souvent un animal ou un enfant (notamment dans $L e$ bruit des choses vivantes) qu'un partenaire masculin.

${ }^{4}$ Bien que l'idée que les animaux aient besoin des humains puisse être choquante, chez Turcotte c'est plutôt que tous les vivants doivent être en relation affective ; sa vision de l'équilibre serait, pour tous, de soigner quelqu'un (humain ou animal) et d'être soigné par quelqu'un (humain ou animal).

${ }^{5}$ L'univers de Turcotte est marqué par la fragilité, comme elle le reconnaît en entretien avec Denise Brassard : «je réalise que tous mes personnages sont sur la corde raide, toujours à la frontière d'un changement de vie, entre deux choses. Ça leur donne une sorte de liberté, malgré tout [...]. Quand on ne sait pas où on est, une liberté de pensée s'installe» (Turcotte à Brassard 17). Cette fragilité des personnages féminins permet une ouverture à l'autre exceptionnelle - notamment à l'autre animal.

${ }^{6}$ Notons que, dans son œuvre, la capacité des animaux à éprouver des émotions ne fait aucun doute.

${ }^{7}$ Le sentiment de n'avoir rien à cacher devant l'oiseau est perçu par la narratrice turcottienne comme une liberté, une occasion privilégiée d'être pleinement soi rarement possible dans la société des hommes. Cette "nudité » émotionnelle qui permet une certaine transparence dans le rapport entre la femme et la perruche contraste vivement avec l'expérience de la nudité physique que Derrida éprouve avec gêne devant le regard de sa chatte (18). Je remercie Rocky Peñate de me l'avoir fait remarquer.

${ }^{8}$ Le traitement par Turcotte de la relation entre le personnage féminin et l'oiseau qu'elle aime est entièrement différent de celui de Flaubert, par exemple, qui présente l'amour de Félicité pour son perroquet Loulou comme ridicule. Il n'y a aucune distanciation ironique chez Turcotte, et l'amour unissant la femme et l'animal est présenté avec tendresse comme une relation éthique basée sur la compassion. Avoir un « cœur simple » permet à la narratrice turcottienne d'entrer en relation avec l'animal de façon authentique.

${ }^{9}$ Si l'on peut parler avec Elisabeth de Fontenay (qui commente ici la pensée de Derrida) de « structure sacrificielle » (1001) dans la société occidentale - qui n'hésite pas à mettre à mort les animaux, entre autres pour les transformer en viande et les ingérer -, le sacrifice animal mis en scène par Turcotte me semble aller dans une autre direction 
puisqu'il n'est pas exigé de la perruche qu'elle se sacrifie pour le bien-être de la famille. Au contraire, son geste est montré comme émanant de la volonté propre de l'animal, qui accomplit un acte de compassion en cherchant à rendre sa mort (inévitable en raison de sa maladie) utile.

${ }^{10}$ L'ouvrage de Haraway The Companion Species Manifesto : Dogs, People, and Significant Otherness a été publié en français sous le titre Manifeste des espèces de compagnie. Chiens, humains et autres partenaires, ce qui ne me semble pas tout à fait équivalent. De même, alors que companion species évoque une égalité entre les partenaires qui sont tous deux compagnes ou compagnons, «espèces de compagnie » me paraît renvoyer de façon plus unidimensionnelle aux animaux en tant que compagnons des humains, sans la relation réciproque qui résonne dans l'expression originelle.

${ }^{11}$ Le tableau de Frida Kahlo (1946) représente un cerf transpercé par neuf flèches ; le sang coule de ses plaies et se répand sur la fourrure. La tête du cerf est remplacée par un autoportrait du visage de Kahlo, sur lequel ont été apposés les oreilles et les bois du cerf. L'animal est dans une clairière près d'une branche cassée, où il apparaît simultanément couché sur le sol et toujours en train de courir, en raison d'un jeu avec la perspective.

${ }^{12}$ Marguerite Duras raconte dans Écrire (46-56) la mort d'une mouche à laquelle elle a assisté en silence mais en l'accompagnant du regard, puis plus tard de l'écriture. Devant le rire de ses proches qui prennent l'anecdote de la mort de la mouche comme une autre preuve de l'excentricité de l'écrivaine, Duras réplique sobrement : «La mort d'une mouche, c'est la mort » (49). Elle ajoute: «L'heure exacte de la mort, consignée, [...] lui donnait une importance d'ordre général, disons une place précise dans la carte générale de la vie sur terre. Cette précision de l'heure à laquelle elle était morte faisait que la mouche avait eu des funérailles secrètes. Vingt ans après sa mort, la preuve en est faite ici, on parle d'elle encore » (50-51).

${ }^{13}$ Remarquons que prêter aux animaux le désir de suicide est encore une fois leur reconnaitre une volonté et une affectivité propres.

${ }^{14}$ Le dessin qui clôt ce texte d'Autobiographie de l'esprit (186) représente « une sirène de terre »: une queue de poisson est surmontée d'un buste et d'une tête de femme. Coiffée de bois de cerfs, la sirène porte des cheveux courts et se tient apparemment immobile, les yeux clos. Les bras, tenus le longs du corps, se terminent par des nageoires et non des mains.

${ }^{15}$ François Paré (37) fait remarquer le «goût marqué pour l'ordinaire et le quotidien » visible dans la poésie de Turcotte; il me semble que son intérêt porté aux animaux familiers relève d'un même registre. Ce qui permet aux narratrices de Turcotte de tenir le coup dans un monde aliénant, c'est bien la compagnie des animaux et des objets familiers, témoins des malheurs et compagnons rassurants dans l'adversité.

${ }^{16}$ Haraway articule cette idée en insistant sur l'importance de reconnaître l'autonomie de l'autre animal, de le voir comme un sujet séparé, mais avec qui on peut établir une relation : "The specific relationality in this kind of regard holds my attention: to have regard for, to see differently, to esteem, to look back, to hold in regard, to hold in seeing, to be touched by another's regard, to heed, to take care of. This kind of regard aims to release and be released in oxymoronic, necessary, autonomy-in-relation. Autonomy as the fruit of and inside relation. Autonomy as trans-acting. » (When Species Meet, 164)

${ }^{17}$ Haraway le formule de la façon suivante : «The question between animals and humans here is, Who are you? and so, Who are we? » (When Species Meet, 208). Cette attitude d'ouverture devant l'autre animal est également une preuve d'humilité, puisque l'humain reconnaît son ignorance : "people must learn to meet dogs as strangers first in order to unlearn the crazy assumptions and stories we all inherit about who dogs are. Respect for dogs demands at least that much » (When Species Meet, 232).

${ }^{18}$ Cette idée est également évoquée chez Haraway lorsqu'elle déforme le commandement « Thou shalt not kill » en «Thou shalt not make killable » (When Species Meet, 80). Par là, elle critique l'attitude humaine selon laquelle tuer un être humain est tabou et criminel, tandis que tuer un être animal est normalisé puisque beaucoup d'animaux sont considérés killable («tuables»), par exemple pour servir de nourriture aux humains ou encore pour des fins de recherche scientifique : "The problem is actually to understand that human beings do not get a pass on the necessity of killing significant others, who are themselves responding, not just reacting. [...] It is not killing that gets us into exterminism, but making beings killable. » (When Species Meet, 80) Dans un tel contexte, reconnaître un visage à un animal empêche qu'il soit considéré tuable. Fontenay fait une observation similaire : «Ce n'est en effet pas à la vie en général qu'il est interdit d'attenter, mais seulement à la vie de l'homme, de l'autre, du prochain » (1001), ce qui place les animaux en position de vulnérabilité. 